\title{
Solution-Phase Racemization in the Presence of an Enantiopure Solid Phase
}

\author{
Cristobal Viedma, ${ }^{*[a]}$ Bastiaan J. V. Verkuijl $1,{ }^{[b]}$ José E. Ortiz, ${ }^{[d]}$ Trinidad de Torres, ${ }^{[d]}$ \\ Richard M. Kellogg, ${ }^{[c]}$ and Donna G. Blackmond $*[b, e]$
}

\begin{abstract}
Solution-phase racemization drives the evolution of single chirality in the solid phase by the "chiral amnesia" process first described by Viedma. The current investigations lay the basis for a better understanding of the mechanism of the solid-phase deracemization by uncoupling the chemical rate processes associated with the intercon-
\end{abstract}

version of enantiomers in the solution phase from the physical processes associated with solution-solid phase transfer via dissolution and reaccretion of

Keywords: amino acids - chirality deracemization - enantioenrichment $\cdot$ racemization molecules onto crystals. In addition, the enantiomer concentration profiles presented in this work, together with an analytical treatment of the racemization process in the presence of excess enantiopure solid, unequivocally reconfirm the validity of the Meyerhoffer double solubility rule for systems under solution racemization conditions.

\section{Introduction}

One of the greatest unsolved problems in chemistry is the origin of homochirality in the biosphere, that is, the fact that $\mathbf{L}$-amino acids and D-sugars dominate in nature, while laboratory experiments produce a racemic mixture unless directed by an added chiral source. ${ }^{[1,2]}$ Several mechanisms de-

[a] Prof. Dr. C. Viedma

Departamento Cristalografia-Mineralogia, Facultad Geologia

Universidad Complutense, 28040 Madrid (Spain)

Fax: $(+34) 913944872$

E-mail: viedma@geo.ucm.es

[b] B. J. V. Verkuijl, Prof. Dr. D. G. Blackmond

Department of Chemistry

Imperial College London, SW7 2AZ London (UK)

[c] Prof. R. M. Kellogg

Syncom B.V., Kadijk 3, 9747 AT Groningen (The Netherlands)

Fax: (+31) 505757399

E-mail: r.m.kellogg@syncom.nl

[d] J. E. Ortiz, T. de Torres

Laboratorio de Estratigrafía Biomolecular

EscuelaTécnica Superior de Ingenieros de Minas de Madrid 28040 Madrid (Spain)

[e] Prof. Dr. D. G. Blackmond

Present address:

Department of Chemistry

The Scripps Research Institute

La Jolla, CA 92037 (USA)

Fax: (+1) 8587842180

E-mail: blackmond@scripps.edu scribing how an initial imbalance between enantiomers might have come about, together with mechanisms for amplifying this imbalance, have been proposed as an explanation for the origin of biomolecular single handedness. ${ }^{[3-12]}$ Most prominent among these are far-from-equilibrium models that include stochastic symmetry breaking coupled with autocatalytic amplification associated with crystallization phenomena, such as Kondepudi's "Eve crystal" model ${ }^{[7]}$ or chemical systems, such as the Soai reaction. ${ }^{[9]}$

Recent intriguing experimental observations of the evolution of solid-phase homochirality for mixtures of $\mathrm{D}$ and $\mathrm{L}$ crystals offer a new model that is distinct from these mechanisms, representing a near-equilibrium interplay between kinetics and thermodynamics in a system that combines physical phase behavior with chemical reaction processes. The original observations were made by Viedma, ${ }^{[11]}$ who demonstrated that a racemic mixture of the two enantiomorphic crystals of the achiral salt $\mathrm{NaClO}_{3}$ in equilibrium with its saturated solution is driven inexorably over time to a single chiral solid, aided by the energy input from abrasive grinding of the crystals. Simultaneously, but independently, Blackmond $^{[13]}$ and Viedma ${ }^{[14]}$ suggested that this phenomenon could be extended to intrinsically chiral compounds that racemize in solution, and this proposal has since been verified experimentally for amino acid derivatives, ${ }^{[15]}$ for the proteinogenic amino acid aspartic acid, ${ }^{[16]}$ and for the amine product of a reversible Mannich reaction. ${ }^{[17]}$

The mechanism of this remarkable process is still under vigorous discussion. ${ }^{[18-21]}$ One of its most compelling features lies in a paradox: the key driving force for the evolution of 
solid-phase homochirality is solution racemization-a process that conventionally erodes chiral discrimination-which in this case provides a conduit between the two chiral solids. Scheme 1 shows how solution racemization allows intrinsi-
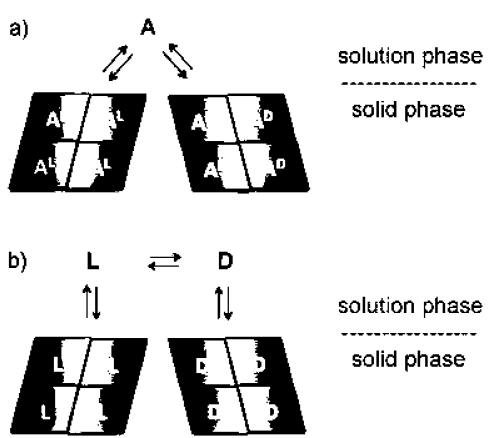

Scheme 1. Solution-solid equilibrium for molecules forming chiral crystals; a) achiral molecule, such as $\mathrm{NaClO}_{3}$; b) chiral molecules forming racemic conglomerate crystals, such as aspartic acid.

cally chiral molecules to lose their solid-phase chiral history and gain the opportunity to choose a new solid-phase chiral destiny in the same way that an achiral molecule, such as $\mathrm{NaClO}_{3}$, does when it dissolves from a chiral crystal. For this reason we have used the term "chiral amnesia" to describe this enantioenrichment process.

Since solution-phase racemization is the key to the process of solid-phase deracemization, our overall mechanistic understanding will be aided by developing a full understanding of this part of the process. A number of puzzling and contradictory experimental observations have been made concerning the relative rates of solution-phase racemization and the overall deracemization process, suggesting that further inquiry is necessary. Kaptein et al. showed that the rate of solid-phase deracemization was proportional to the concentration of the base used to catalyze solution phase racemization. ${ }^{[22]}$ This is curious because solution phase racemization proceeds much more rapidly than the overall deracemization process, suggesting that the solution reaction should not be rate determining. In contrast to this case, Tsogoeva and colleagues noted that the solution racemization rate appeared to be significantly slower than the solid phase deracemization procecss. ${ }^{[17 b]}$ They invoked participation of the solid in the racemization process to rationalize this result.

We report here solubility and enantiomeric excess measurements in two different systems that help to uncouple the solution chemical reaction from the physical processes of dissolution/recrystallization. Our results show that the effect of the presence of the solid phase on solution-phase racemization can be described without the need to invoke special interactions at the solid-solution interface in these systems. Further, we show that changes in solution phase $e e$, which is a relative measurement, may be misleading unless they take into account the absolute change in each enantiomer concentration. This work also reconfirms the validity of the Meyerhoffer double solubility rule ${ }^{[23]}$ for conglomerate systems under racemizing conditions.

\section{Results and Discussion}

The rate of racemization was studied for the two systems shown in Scheme 2, aspartic acid (1) and $\mathrm{N}$-(2-methylbenzylidene)-phenylglycine amide (2), both of which are compounds that we have previously shown undergo the "chiral amnesia" process. ${ }^{[15,16]}$ We monitored absolute concentration profiles of both enantiomers as well as solution phase ee under two sets of conditions. In one case, we followed racemization of the homogeneous enantiopure solution in which the total number of solution phase molecules is fixed (Scheme $3 \mathrm{a}$ ); in the other case we studied solution phase racemization in the presence of varying amounts of the enantiopure solid phase in equilibrium with the solution phase, which thus provides a path for either addition or removal of molecules to or from the solution phase (Scheme $3 \mathrm{~b}$ ).

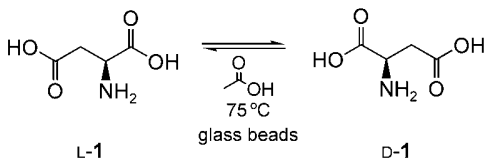

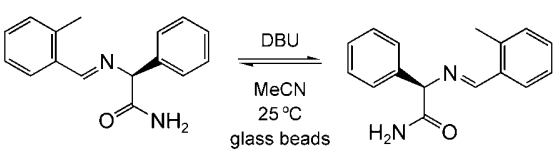

Scheme 2. Systems employed in studying solution-phase racemization and solid-phase deracemization.

Plots of $\ln (e e)$ versus time are commonly used to measure the solution phase racemization rate constant. ${ }^{[24]}$ Figure 1 shows results for the racemization of aspartic acid L-1 carried out in the presence of varying amounts of the solid phase Lamino acid. The rate of change of solution phase $e e$ shows a clear dependence on the amount of solid initially present. ${ }^{[25]}$ In addition, for lower initial amounts of solid the slope increases by approximately a factor of two at longer racemization times, and it was observed that less solid remained at the end of the experiment than at the beginning.

A similar effect of the amount of solid was seen in racemization of the Schiff base system of Scheme $2 b$ in the presence of enantiopure solid 2 . The change in solution phase $e e$ as a function of time was found to be approximately a factor of two lower in the presence of solid than it is for racemization in the homogeneous solution. Decreasing the amount of solid ultimately resulted in a rate of change of solution phase ee equaling that measured in homogeneous solution in the absence of solid (Figure 2). 


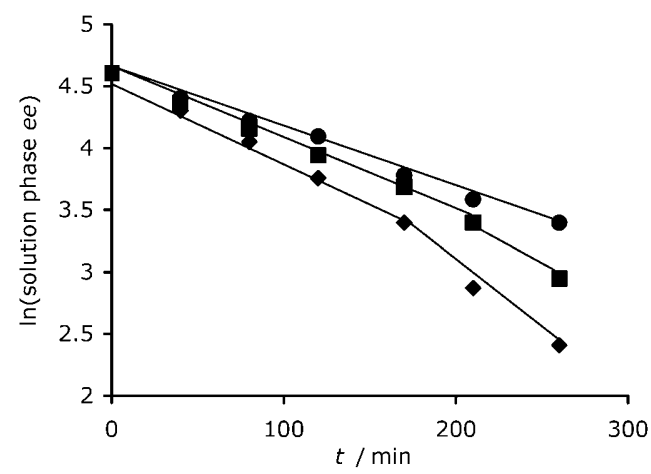

Figure 1. Log plot of the change in solution phase $e e$ over time as a function of the amount of solid aspartic acid L-1 $(\bullet: 0.8 \mathrm{~g}, \mathbf{m :} 0.5 \mathrm{~g}, \bullet: 0.2 \mathrm{~g})$ in acetic acid at $75^{\circ} \mathrm{C}$ (Scheme $\left.2 \mathrm{a}\right)$.

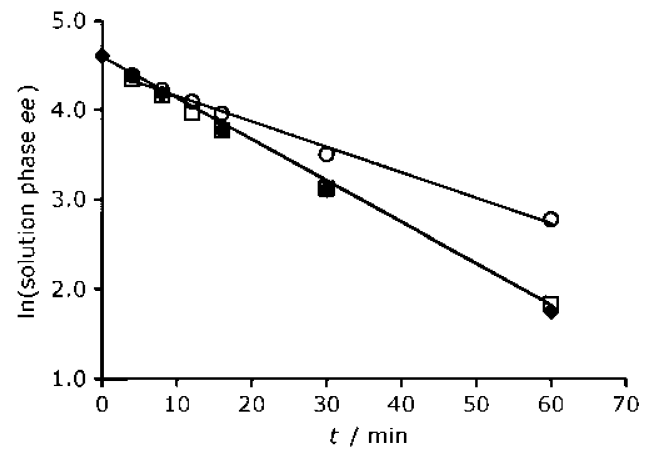

Figure 2. Log plot of the change in solution phase ee over time as a function of the amount of solid $N$-(2-methylbenzylidene)-phenylglycine amide in MeCN with DBU (Scheme 2b); DBU concentration was $2 \mathrm{~mm}$.

These results appear to suggest that the rate of racemization in solution is dependent on the amount of solid present in the system, giving rise to speculation about mechanisms through which the solid phase might play a role in the solution racemization process. However, under closer examination of the racemization process, we find that a different picture emerges.

Monitoring the absolute concentrations of enantiomers of $\mathbf{2}$ in solution as racemization proceeds, provides the data shown in Figure 3 for the system of Scheme 2 b. Solution concentrations over the time course of racemization for a system containing a large excess of enantiopure solid $(S)$-2 were compared with those in a homogeneous saturated solution of $(S)$ 2. In the case of the homogeneous solution, Figure 3 a shows that the $(S)$-2 concentration in solution decreases while the
(R)-2 concentration rises-behavior that is characteristic of racemization in homogeneous solution in which the sum of $((R)-\mathbf{2}+(S)-2)$ in solution is necessarily constant over the course of the racemization. In the case of a large excess of solid, however, we observe that while the $(R)$-2 concentration rises as expected during racemization, the concentration of $(S)$-2 does not decrease, but remains essentially constant over time (Figure $3 \mathrm{~b}$ ). This results in an overall increase-in fact a doubling - of the total concentration of enantiomers $((R)-\mathbf{2}+(S)-\mathbf{2})$ in solution when the system is fully equilibrated. Thus, the solid phase rapidly replenishes the solution phase with $(S)$-2 molecules to compensate for the loss of those that are converted to $(R)-2$ molecules via racemization. Figure 3 thus presents clear kinetic evidence of the validity of the Meyerhoffer double solubility rule in the case of racemizing enantiomers.

Our observation of a factor of two difference in the slope of plots of $\ln (e e)$ versus time for racemizing homogeneous solution compared to systems containing excess solid deserves further discussion. The results of Figure 3 show similar initial rates of increase in solution phase $(R)-2$ concentration, indicating that the rate of conversion from one enantiomer to the other in solution does not depend on the presence of solid. Solution $e e$, however, is a function of the absolute concentrations of both $R$ and $S$. Because the absolute temporal solution concentrations of $R$ and $S$ are influenced differently depending on whether solid is present or absent, the interpretation of the change in solution enantiomeric excess becomes more complicated. Solution enantiomeric excess profiles for the two cases with enantiopure solid present, in which only one enantiomer concentration is changing, and in homogeneous solution, in which both enantiomer concentrations are changing, cannot be directly compared as a means to monitor racemization rate.

We may rationalize the observed $e e$ profiles through an analytical treatment of the rate of solution racemization for the two cases shown in Figure 3. In the case of racemization in a homogeneous solution as shown in Equation (1), where
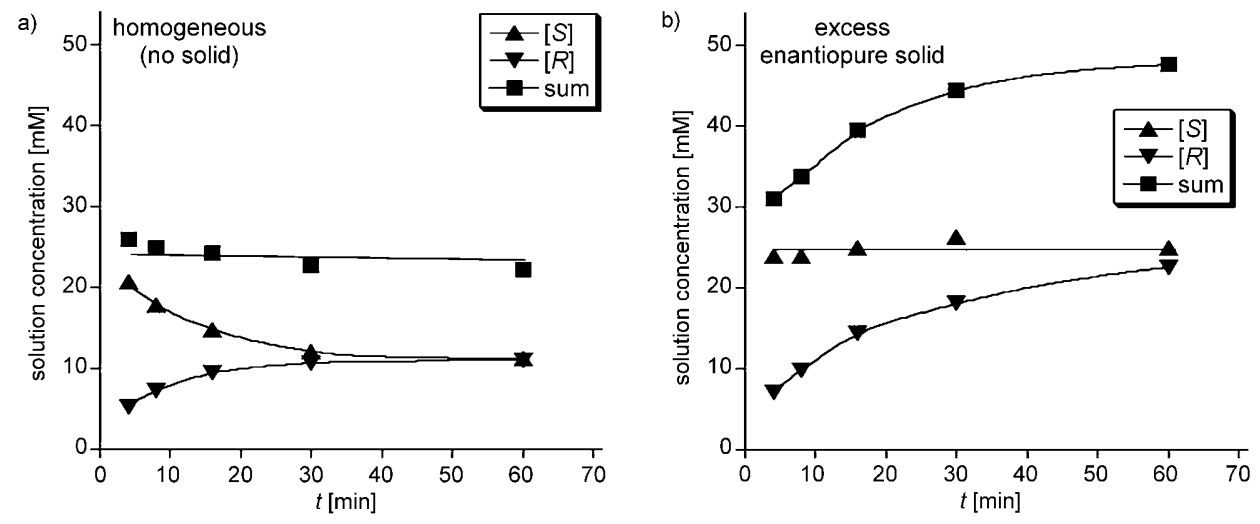

Figure 3. Time-course profiles for the absolute solution concentrations during racemization of the amino acid derivative 2 (Scheme 2) at ambient temperature starting with saturated solutions of enantiopure $(S)$-2 in: a) homogeneous solution; and b) the presence of excess solid $(S)-\mathbf{2}$. $\mathbf{\Delta}$ : solution concentration of $(S)$-2; $\mathbf{\nabla}$ : solution concentration of $(R)-\mathbf{2}$; $\mathbf{m}$ : sum of the solution concentrations of the two enantiomers (total: $(R)-\mathbf{2}+(S)$ 2). DBU solution concentration was $6 \mathrm{~mm}$ in both cases. 
the total concentration of $[R]+[S]$ remains constant, plots of $\ln (e e)$ versus time are routinely employed to measure the

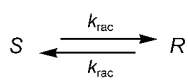
racemization rate constant for

(1) the case in homogeneous solution. ${ }^{[24,26]}$

Such plots derive from the solution of the first order linear differential Equation (2) describing the increase in solution concentration of $R$ as racemization proceeds in an enantiopure solution of concentration $[S]_{0}$, where $[R]+[S]=$ constant $=[S]_{0}$ and $k_{\text {rac }}$ is the forward and reverse racemization rate constant.

$\frac{\mathrm{d}[R]}{\mathrm{d} t}=k_{\mathrm{rac}}[S]-k_{\mathrm{rac}}[R]=k_{\mathrm{rac}}\left([S]_{0}-[R]\right)-k_{\mathrm{rac}}[R]$

$\frac{\mathrm{d}[R]}{\mathrm{d} t}+2 k_{\mathrm{rac}}[R]=k_{\mathrm{rac}}[S]_{0}$

The analytical solution of this equation, employing the initial condition of enantiopure solution with $[S]=[S]_{0}$ and $[R]=0$ at $t=t_{0}$, is given by Equations (3a) and (3b) to describe the $R$ and $S$ concentrations as a function of time.

$[R]=\frac{[S]_{0}}{2}\left(1-e^{-2 k_{\mathrm{rac}} t}\right)$

$[S]=\frac{[S]_{0}}{2}\left(1+e^{-2 k_{\mathrm{rac}} t}\right)$

Substituting these relationships into the equation for enantiomeric excess, defined with $[S]$ excess as positive, gives Equation (4) for $e e$ as a function of time during the racemization process. Thus, the slope of a plot of $\ln (e e)$ versus time is given by $2 k_{\mathrm{rac}}$.

$\% e e=100 \frac{[S]-[R]}{[S]-[R]}=100 e^{-2 k_{\text {rac }} t}$

$\ln (\% e e)=-2 k_{\mathrm{rac}} t+4.6$

However, when racemization is carried out in the presence of the enantiopure solid, these relationships can no longer be employed. As our data show, and as the Meyerhoffer double solubility rule predicts, the total quantity $[R]+[S]$ in solution is no longer constant. As racemization proceeds, the solution concentration increases over time as the loss of $S$ by conversion to $R$ is compensated for by dissolution of $S$ from the $S$ solid. For the case of a large amount of solid, we may assume that replenishment is rapid and the solution concentration of $S$ remains constant at $[S]_{0}$ over the course of the racemization, as was found for the case shown in Figure $3 \mathrm{~b}$. This provides a new differential equation describing the racemization [Eq. (5)]. This equation may be solved by using the same initial conditions as previously employed, giving Equation (6) for the temporal concentrations of $R$ and $S$ enantiomers.

$\frac{d[R]}{d t}=k_{\mathrm{rac}}[S]_{0}-k_{\mathrm{rac}}[R]$
$\frac{d[R]}{d t}+k_{\mathrm{rac}}[R]=k_{\mathrm{rac}}[S]_{0}$

$[R]=[S]_{0}\left(1-e^{-k_{\mathrm{rac}} t}\right)$

$[S]=[S]_{0}$

From Equation (6), the relationship for solution enantiomeric excess as a function of time during racemization in the presence of excess solid is given by Equation (7).

$$
\begin{aligned}
& \% e e=100 \frac{[S]-[R]}{[S]+[R]}=\frac{e^{-k_{\mathrm{rac}} t}}{2-e^{-k_{\mathrm{rac}} t}} \\
& \ln (\% e e)=-k_{\mathrm{rac}} t-\ln \left(2-e^{-k_{\mathrm{rac}} t}\right)+4.6=-k_{\mathrm{rac}} t+\approx 4.0
\end{aligned}
$$

Thus, it may be seen by comparing Equations (4) and (7), the initial rate of change in solution ee as a function of time will be decreased by about twofold when racemization is carried out in the presence of an excess of the enantiopure solid in rapid exchange with its solution phase. This finding is a consequence of the combination of the Meyerhoffer double solubility rule, which tells us that molecules will be driven from the solid to the solution phase during racemization so that $[R]+[S] \neq[S]_{0}$, with the fact that solution-solid phase transfer is rapid compared to solution racemization, which results in $[S]=[S]_{0}$ throughout the process. Plots of $\ln$ (solution $e e$ ) versus time for systems under these conditions will approach a twofold difference in slope for racemization in the presence of a solid phase compared to homogeneous solution. Figure 4 shows the general case in theoretical plots of $\ln (e e)$ versus the function $\left(k_{\mathrm{rac}} t\right)$ on the $x$ axis for Equations (4) and (7).

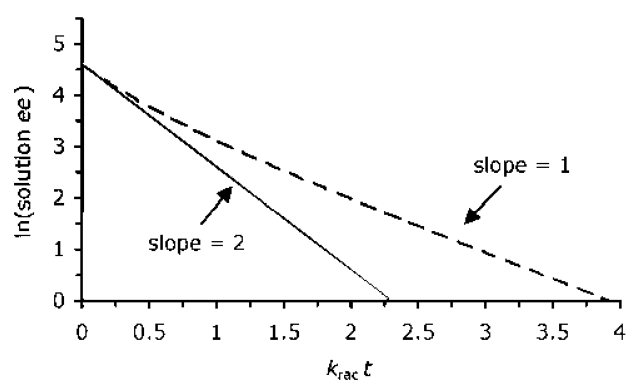

Figure 4. Plot of $\ln$ (solution $e e)$ versus time as a function of $\left(k_{\mathrm{rac}} t\right)$ for the case of a homogeneous solution (-) according to Equation (4) and in the presence of a solid phase (----) according to Equation (7).

The experimental confirmation of Equations (4) and (7) was shown in Figure 2, where the difference in slope for racemization in homogeneous solution by using $2 \mathrm{~mm}$ DBU (slope $=0.045 \mathrm{~min}^{-1}$ ) compared to racemization in the presence of a solid (slope $=0.028 \mathrm{~min}^{-1}$ ) is an apparent effect due to the presence of the solid; the intrinsic kinetic racemization rate constant remains unchanged, and the chemical process of racemization continues to be described solely by 
solution phase interactions even in the presence of the solid phase.

These findings may be considered in the context of experimental observations that have been made for different systems that undergo the chiral amnesia process. One clear conclusion is that the rationale of surface-aided racemization invoked by Tsogoeva for their Mannich system does not apply to either aspartic acid (1) or phenylglycine amide Schiff base 2 studied in this work, since the presence of the solid has no effect on intrinsic racemization rate in these systems. Our work also indicates that the enhancement in overall deracemization rate for $\mathbf{2}$ that has been observed with increasing [DBU] cannot be explained simply by a catalytic effect on solution racemization, since solid-solution equilibration is much more rapid than solution racemization, and solution racemization is much faster than the overall deracemization process at all levels of DBU. These studies, which involve uncoupling the rates of the different physical and chemical processes, will aid us in developing the detailed mechanistic understanding of the chiral amnesia process that continues to elude us.

\section{Conclusion}

Careful measurements of absolute concentrations of solution-phase enantiomers during racemization both under homogeneous conditions and in the presence of excess enantiopure solid in equilibrium show that the rate of change of solution phase ee will be lower by twofold for the former case. This experimental result is rationalized by a theoretical treatment and confirms the validity of the Meyerhoffer double solubility rule under conditions of solution racemization. The intrinsic racemization rate constant is shown to be identical in the presence and absence of the solid phase for the phenylglycineamide Schiff base 2, which is the first intrinsically chiral compound shown to undergo solid phase deracemization via the "chiral amnesia" process. No special interactions between solution and solid phases need be invoked to explain these observations.

\section{Experimental Section}

Chemicals: $(S)-N$-(2-Methylbenzylidene)-phenylglycine amide was kindly provided by DSM Pharma and Syncom. DBU and aspartic acid were obtained from Aldrich. Acetonitrile was obtained at Riedel-de Haën, dried prior to use with $\mathrm{Na}_{2} \mathrm{SO}_{4}$ and stored over $4 \AA$ mol sieves under Argon atmosphere.

Experimental procedure for aspartic acid (1): Solution-solid mixtures of $\mathrm{L}$-aspartic acid at various amounts $(0.2,0.5,0.8 \mathrm{~g}$ in $8 \mathrm{~mL}$ of acetic acid) were magnetically stirred $(900 \mathrm{rpm})$ for four days at ambient temperature in the presence of $1 \mathrm{~mm}$ glass beads $(6 \mathrm{~g})$ forming a slurry. After establishing solution-solid equilibrium, solution-phase racemization was initiated in the absence of glass beads by adding salicylaldehyde $(0.05 \mathrm{~mL})$ as catalyst at room temperature $\left(25^{\circ} \mathrm{C}\right)$ in some experiments and by increasing the temperature to $75^{\circ} \mathrm{C}$ without salicylaldehyde in other experiments. Samples were collected over time and centrifuged to separate the solid phase. Solution phase enantiomeric excess was measured by using chiral HPLC methods as previously described. ${ }^{[16]}$

Experimental procedure for $\boldsymbol{N}$-(2-methylbenzylidene)-phenylglycine amide (2): Two reaction mixtures were prepared, the first (a) with $(S)$ Schiff base $(50 \mathrm{mg})$ in acetonitrile $(1.5 \mathrm{~mL})$ and the second $(\mathbf{b})$ with $(S)$ Schiff base $(50 \mathrm{mg})$ in acetonitrile $(1.0 \mathrm{~mL})$. A magnetic stirrer and $1.0 \mathrm{~g}$ of glass beads $(2.5 \mathrm{~mm})$ were added and the reaction mixtures were stirred, overnight. Both reaction mixtures were white suspensions. Reaction mixture (a) was taken up in a syringe and filtered over a $0.45 \mu \mathrm{m}$ filter and $1.0 \mathrm{~mL}$ of the filtrate was put in a new flask. A stock solution of DBU $(60.0 \mathrm{~mm})$ in acetonitrile was prepared and $0.1 \mathrm{~mL}$ of stock solution was added to both reaction mixtures. The mixtures were stirred vigorously and the conversion was monitored over time by taking samples. The absolute concentrations of the Schiff base enantiomers were determined according to a method previously described. ${ }^{[20]}$

\section{Acknowledgements}

D.G.B. acknowledges funding from Merck \& Co. and a Royal Society Wolfson Research Merit Award. C.V. acknowledges helpful discussions with members of the European COST Action CM0703 "Systems Chemistry".

[1] W. A. Bonner, Origin Life Evol. Biosphys. 1991, 21, 59.

[2] J. L. Bada, Nature 1995, 374, 594.

[3] F. C. Frank, Biochim. Biophys. Acta 1953, 11, 459

[4] M. Calvin, Molecular Evolution, Oxford University Press, Oxford, 1969.

[5] a) E. Havinga, Chem. Weekbl. 1941, 38, 642; b) E. Havinga, Bio chim. Biophys. Acta 1954, 13, 171.

[6] a) I. Weissbuch, L. Addadi, L. Leiserowitz, M. Lahav, J. Am. Chem. Soc. 1988, 110, 561; b) I. Weissbuch, L. Leiserowitz, M. Lahav, Tetrahedron 1987 43, 1281; c) I. Weissbuch, L. Leiserowitz, M. Lahav, Top. Curr. Chem. 2005, 259, 123.

[7] D. K. Kondepudi, R. J. Kaufman, N. Singh, Science 1990, 250, 975.

[8] H. Morowitz, J. Theor. Biol. 1969, 25, 491-494.

[9] K. Soai, T. Shibata, H. Morioka, K. Choji, Nature 1995, 378, 767

[10] D. G. Blackmond, Proc. Natl. Acad. Sci. USA 2004, 101, 5732.

[11] C. Viedma, Phys. Rev. Lett. 2005, 94, 065504.

[12] M. Klussmann, H. Iwamura, S. P. Mathew, D. H. Wells, Jr., U. Pandya, A. Amstrong, D. G. Blackmond, Nature 2006, 441, 621.

[13] D. G. Blackmond, Chem. Eur. J. 2007, 13, 3290.

[14] C. Viedma, Astrobiology 2007, 7, 312

[15] W. L. Noorduin, T. Izumi, A. Millemaggi, M. Leeman, H. Meekes, W. J. P. van Enckevort, R. M. Kellogg, B. Kaptein, E. Vlieg, D. G. Blackmond, J. Am. Chem. Soc. 2008, 130, 1158.

[16] C. Viedma, J. E. Ortiz, T. de Torres, T. Izumi, D. G. Blackmond, $J$. Am. Chem. Soc. 2008, 130, 15274.

[17] a) S. B. Tsogoeva, S. Wei, M. Freund, M. Mauksch, Angew. Chem. 2009, 121, 598; Angew. Chem. Int. Ed. 2009, 48, 590; b) S. Wei, M Mauksch, S. B. Tsogoeva, Chem. Eur. J. 2009, 15, 10255.

[18] a) J. Crusats, S. Veintemillas-Verdaguer, J. M. Ribó, Chem. Eur. J. 2006, 12,7776 ; b) J. Crusats, S. Veintemillas-Verdaguer, J. M. Ribó, Chem. Eur. J. 2007, 13, 10303.

[19] D. G. Blackmond, Chem. Eur. J. 2007, 13, 10306

[20] T. Izumi, D. G. Blackmond, Chem. Eur. J. 2009, 15, 3065.

[21] For a general comment see the "News and Views" article: J. M. McBride, J. M. Tully, Nature 2008, 452, 161.

[22] W. L. Noorduin, H. Meekes, W. J. P. van Enckevort, A. Millemaggi, M. Leeman, B. Kaptein, R. M. Kellogg, E. Vlieg, Angew. Chem. 2008, 120, 6545; Angew. Chem. Int. Ed. 2008, 47, 6445.

[23] W. Meyerhoffer, Ber. Dtsch. Chem. Ges. 1904, 37, 2604.

[24] E. Eliel, S. H. Wilen, Stereochemistry of Organic Compounds, John Wiley \& Sons, New York, 1994, pp. 424-426. 
[25] In all solution racemization experiments carried out in the presence of the enantiopure solid, the solid phase remains enantiopure while the solution phase undergoes racemization. This has been shown previously (see refs. $[15,16]$ ) and is attributed to the fact that primary nucleation to form the first crystals of the enantiomeric solid is a much more activated process and is highly disfavored under these conditions.
[26] The mathematical derivation of enantiomeric excess as a function of time during racemization in homogeneous solution that is given in Equations (2)-(4) is derived in ref. [24], however, repeated here in order to contrast this with the expressions obtained for racemization in the presence of enantiopure solid, which to our knowledge have not been presented previously. 